ISSN: 2637-7632

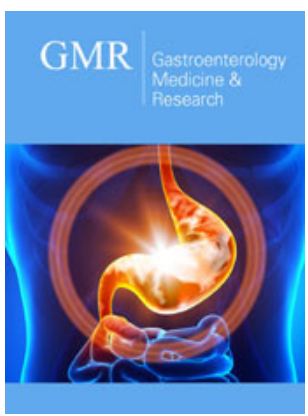

*Corresponding author: Farid Gharagozloo, Center for Advanced Thoracic Surgery, Global Robotics Institute, Advent health Celebration, University of Central Florida, USA

Submission: 海June 25, 2021

Published: 啙July 08, 2021

Volume 6 - Issue 1

How to cite this article: Farid Gharagozloo. Laparoscopic Modified Belsey Fundoplasty (Gastroesophageal Valvuloplasty) for relief of Gastroesophageal Reflux Disease: Review of Technique and Results. Gastro Med Res. 6(1). GMR. 000627. 2021.

DOI: 10.31031/GMR.2021.06.000627

Copyright@ Farid Gharagozloo, This article is distributed under the terms of the Creative Commons Attribution 4.0 International License, which permits unrestricted use and redistribution provided that the original author and source are credited.

\section{Laparoscopic Modified Belsey Fundoplasty (Gastroesophageal Valvuloplasty) for relief of Gastroesophageal Reflux Disease: Review of Technique and Results}

\author{
Farid Gharagozloo* \\ Center for Advanced Thoracic Surgery, University of Central Florida, USA
}

\begin{abstract}
Nissen fundoplication is by far the most common surgical procedure for the repair of gastroesophageal reflux in the United States. However, Nissen fundoplication is associated with significant complications and a high failure rate. Belsey Mark IV repair creates a valve spanning 270 of the circumference of the gastroesophageal junction by intussuscepting the esophagus into the stomach by $2 \mathrm{~cm}$. However, the conventional Belsey Mark IV repair as performed through a left thoracotomy is associated with the morbidity of a thoracotomy. A laparoscopic Belsey repair is a minimally invasive procedure which is associated with excellent relief of reflux. We review the technique and results of laparoscopic Belsey fundoplasty.
\end{abstract}

Keywords: GERD; Fundoplication; Belsey; Antireflux surgery; Gastroesophageal valvuloplasty

\section{Introduction}

Gastroesophageal Reflux Disease (GERD) affects approximately 20\% of Americans and is the most commonly diagnosed disease of the upper Gastrointestinal (GI) tract in humans [1,2]. Pathologic reflux is associated with esophageal carcinoma [3]. Curiously, despite greater use of acid suppressive therapy esophageal adenocarcinoma has increased by $660 \%$ in frequency in the United States since the 1970's [4,5]. Therefore, even with the increase in the use of proton pump inhibitors, the advent of minimally invasive procedures and the promise of prevention of metaplastic and dysplastic changes in the lower esophagus with surgery have resulted in greater interest in a physiologic surgical procedure which would restore the normal antireflux barrier.

\section{Nissen fundoplication versus belsey mark IV fundoplasty}

Nissen fundoplication is the most common surgical procedure which is advocated for gastroesophageal reflux. Nissen fundoplication increases the lower esophageal sphincter pressure or high-pressure zone significantly more than a partial fundoplication. However, Nissen fundoplication is associated with significant dysphagia, gas bloat and unpredictable longevity [6]. There are reports of up to $40 \%$ for dysphagia and $31 \%$ the inability to vomit following Nissen fundoplication [6-8]. The Belsey Mark IV repair intussuscepts the esophagus into the stomach by $2 \mathrm{~cm}$ for 270 degrees of the GE junction thereby creating a valve. Long term follow up of patients undergoing Belsey Mark IV has shown good-to-excellent results in $78-95 \%$ of patients. Even though the Belsey Mark IV repair is as effective as the Nissen fundoplication for the relief of reflux, it is associated with a significantly lower rate of postoperative dysphagia and gas bloat syndrome. After Belsey mark IV Fundoplication, gas bloat has been reported in 3\% and dysphagia in 3\% of patients [9]. However, the conventional 
Belsey Mark IV repair as performed through a left thoracotomy is associated with a number of disadvantages. Major disadvantages of the Belsey Mark IV procedure are:

A. It is a conceptually a more complex procedure and is more difficult to teach.

B. Location, depth, and spacing of each suture for the repair is crucial to favorable results.

C. The Belsey Mark IV repair is performed through left thoracotomy with the attendant morbidity.

D. Until recently, a laparoscopic Belsey procedure has not been possible.

\section{Belsey fundoplasty and the gastroesophageal antireflux mechanism}

A significant component of gastroesophageal antireflux mechanism is the highly complex three-dimensional relationship which exists between the gastroesophageal junction and the esophageal hiatus [10-12]. The gastroesophageal valve is the $2 \mathrm{~cm}$ Musculo mucosal fold which extends from the greater curve to the lesser curve of the stomach and is created by the oblique intussusception of the esophagus into the stomach. In turn, the gastroesophageal valve is suspended on the esophageal hiatus by the phrenoesophageal ligament. Suspension of the valve onto the esophageal hiatus prevents kinking and incompetence of the valve in the normal setting. With a hiatal hernia and stretching of the phrenoesophageal ligament, the esophagus gets pulled out of the stomach much like a telescope being extended and the Musculo mucosal fold disappears, resulting in GERD. Curiously through rigorous observation and trial and error, Belsey designed a procedure that would recreate what is presently considered to be the normal antireflux barrier. The Belsey Mark IV Fundoplasty (BF) procedure recreates the gastroesophageal antireflux mechanism by intussuscepting the esophagus into the stomach, thereby creating a gastroesophageal valve and suspending it onto the esophageal hiatus. In a porcine model of GERD, the BF has been shown to most closely recreate the normal antireflux mechanism [13].

\section{Technique of laparoscopic modified belsey fundoplasty (gastroesophageal valvuloplasty)}

The patient is placed in the lithotomy position (Figure 1). The surgeon stands between the legs. Two laparoscopic $\mathrm{CO}_{2}$ insufflators are used. Port placement is similar to Nissen fundoplication. Port 1 is placed at the umbilicus. A 0-degree Endo eye video endoscope (Olympus Inc.) is used. Pneumoperitoneum is created using $\mathrm{CO}_{2}$ gas to a maximum pressure of $15 \mathrm{mmHg}$. The table is placed in a reverse Trendelenburg position. Under direct videoendoscopic guidance, four other ports are placed. The 10-12 Vertiport trocar (Covidien/Medtronic Inc., Norwalk, Conn.) is used for all ports. A design advantage of these ports is that the port sites do not have to be closed. Port 2 is placed in the right paraumbilical region at the mammary line. An Endo-Paddle Retractor (Medtronic Inc., Norwalk, Conn.) is introduced through this Port and fixed to the table using a self-retaining clamp system (Medifex, Velmed Inc.,
Wexford, Penn). Port 3 is placed in the Para umbilical region in the left mammary line. A second Endo-paddle retractor is introduced through Port 3 and used to retract the stomach. Port 4 is placed in the subcostal region halfway between the umbilicus and the xiphoid just to the left of the midline. This port is aligned with the right limb of the right crus of the diaphragm. Port 5 is placed in the subcostal region two finger-breadths to the left and caudad to Port 4. Port 5 is aligned with the left limb of the right crus of the diaphragm. The laparoscopic insufflator is disconnected from Port 1 and attached to Port 4. A second insufflator is attached to Port 5. The use of two high-flow insufflators facilitates rapid extra corporeal knot placement while preserving pneumoperitoneum and exposure of the esophageal hiatus.

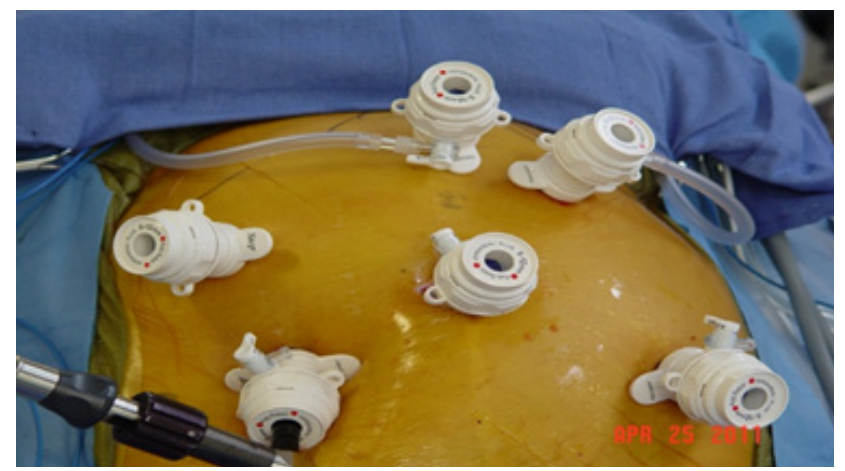

Figure 1: Port placement.

A 30-degree Endo eye video endoscope is used for the remainder of the procedure. An endo grasper is introduced through Port 4, and endo shears with cautery attachment is introduced through Port 5. The right crural arch is identified. The phrenoesophageal ligament is divided. The hepatogastric momentum is divided and the caudate lobe of the liver is identified. At this point, the Right Limb (RL) of the right crus is visualized. The lateral and medial borders of the RL are identified. The endoscopic Endo paddle retractor is placed between the RL and the esophagus and used to provide lateral traction to the esophagus. The fatty tissue overlying the RL is excised, and the RL is followed inferiorly to its junction with the Left Limb (LL) of the right crus. Next, the dissection of the RL is carried superiorly onto the crural arch and around to the LL of the right crus. The LL is dissected inferiorly by taking down the angle of His and gastric fundal attachments. Lateral traction on the paddle retractor moves the esophagus laterally to the left and facilitates the exposure of the entire crural sling. The " $\mathrm{V}$ "-shaped junction between the right limb and the left limb of the right crus of the diaphragm is visualized. This facilitates exposure of the aorta which traverses posterior and deep to this junction through the left diaphragmatic crural sling. Importantly, encirclement of the esophagus or division of the short gastric vessels is not required.

\section{Posterior crural closure}

Posterior crural closure is accomplished by re-approximating the RL and LL with two to three sutures. We prefer the Endo Stitch instrument (Medtronic Inc.) with 0 Ethibond suture (Figure 2). When approximating the RL and LL of the right crus posterior, the 
straight needle of the Endo Stitch instrument passes in a tangential plain anterior to the aorta and carries a lower risk of inadvertent aortic injury which usually is the result of deep suture placement with a curved needle. A $1 \mathrm{~cm}$ square absorbable pledget cut from Vicryl mesh (Ethicon, Inc.) is passed through Port 4. The Endo Stitch with 0 Ethibond is passed through Port 5. Intracorporeally the pledget is loaded onto the needle. The needle is passed through LL and RL, respectively. Next, Intracorporeally the needle is passed through a second vicryl pledget which is introduced with the grasper in the surgeon's left hand. The Endo stitch carrying the suture is withdrawn out of the entry Port 5, and extracorporeal knots are placed using a long external knot pusher. The suture is cut above the knot. This technique is repeated for all the posterior crural sutures.

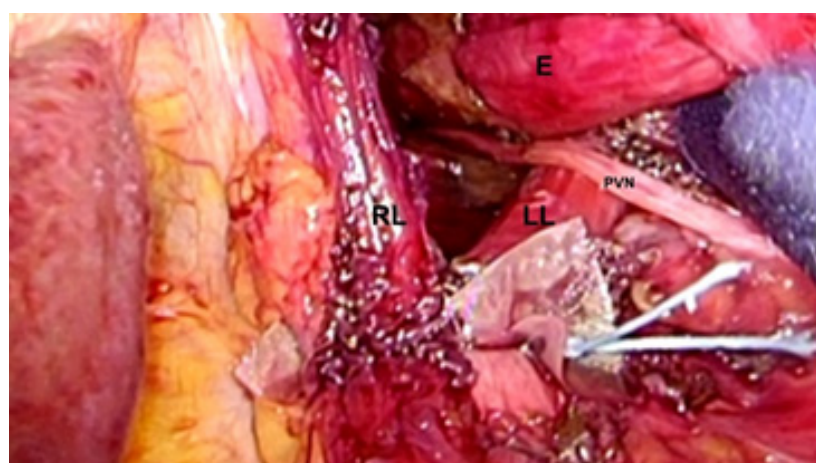

Figure 2: The esophagus $€$ is retracted laterally and the posterior aspect of the Right Limb (RL) and the Left Limb (LL) are re-approximated with 0 Ethibond with Vicryl pledgets.

\section{Anterior crural closure}

In a similar manner to the posterior crural closure, 0 Ethibond sutures on the Endo stitch instrument with intracorporeally loaded pledgets of vicryl mesh are used to reapproximate the anterior portion of the crural arch. This step represents a modification of the original Belsey Mark IV technique. However, in our experience, the anterior crural closure allows for the formation of an acute angle at the gastroesophageal junction and recreates one of the important features of the normal antireflux barrier. The sutures are passed through Port 5, a vicryl pledget is loaded on the suture Intracorporeally, and the suture is passed through the RL and LL of the crural arch. A second vicryl pledget is loaded Intracorporeally onto the suture, and the suture is tied using extra corporeal technique as outlined previously. Usually, one to two anteriorly placed sutures are required. The crural closure is sized based on the passage of a 60 -French bougie into the distal esophagus. Following crural closure, the Belsey fundoplasty is performed.

\section{Belsey fundoplasty}

The intussusception of the esophagus into the stomach is accomplished for the anterior 270 degrees (from RL to LL of the right crus) of the 360-degree circumference of the esophagogastric junction (Figure 3). The esophagogastric fat pad is removed. When looking at the esophagus directly, position of the sutures is determined by the face of a clock. The esophagus is marked $2 \mathrm{~cm}$ above the esophagogastric junction (EG) at the 3 o'clock position lateral to left vagus nerve (E1), at the 9 o'clock position just in front of the right vagus nerve (E3), and halfway in between at approximately the 11 o'clock position (E2). The stomach is marked $2 \mathrm{~cm}$ below the GE junction at the greater curvature (G1), the lesser curvature (G3), and at a point halfway between G1 and G3 (G2). The Endo stitch instrument with 00 Ethibond is introduced through Port 5. The first Belsey suture (E1 to G1, Greater Curve) passes in a mattress fashion from G1 to E1 and through the diaphragm at the left crural limb (Figure 4-6). A vicryl pledget is introduced with a grasper through Port 4. The suture is withdrawn through Port 5. Metal clips are placed on the free ends of the suture in order to facilitate identification and recovery of the suture at a later point. The untied suture is reintroduced through Port 5 and deposited in the left upper quadrant away from the GE junction. This suture is tied a later time. Placing a tie on the "G1-E1" suture at this time will obscure the precise placement of the "E2-G2" and "E3- G3" sutures. The second Belsey suture (E3 to G3, Lesser curve) is passed in a similar manner from G3 to E3 and onto the diaphragm at the right crural limb. Similar to the greater curvature suture, this suture is withdrawn through Port 5, tagged with metal clips and deposited in the right upper quadrant away from the GE junction. This suture will be tied a later time (Figure 7-10).

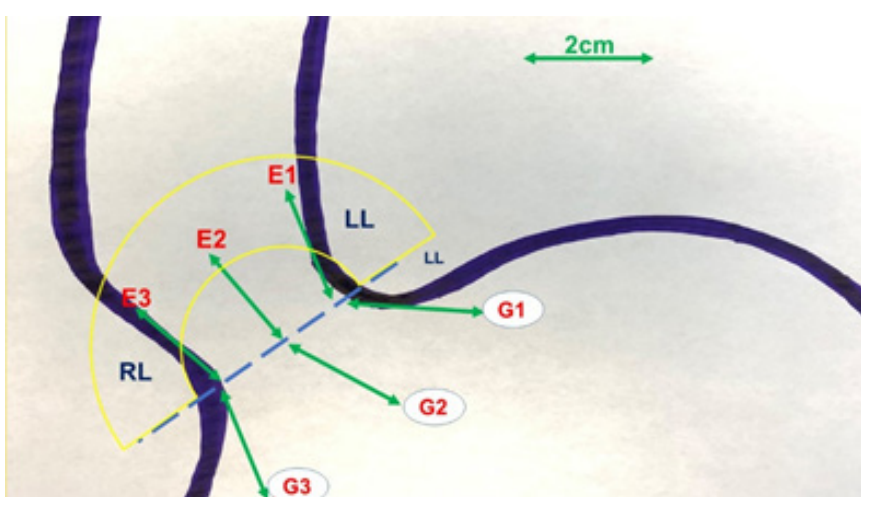

Figure 3: Pattern of suture placement for the Belsey Repair. $\mathrm{E}=$ Esophagus, $\mathrm{G}=$ Stomach, $\mathrm{RL}=$ Right limb of the crus, LL = Left limb of the crus.

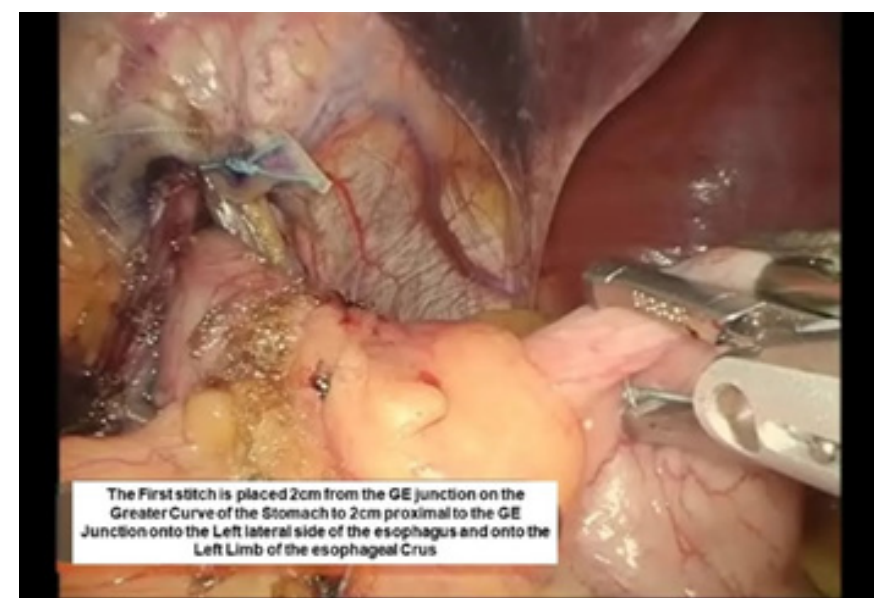

Figure 4: First belsey suture placement G1 to E1. 


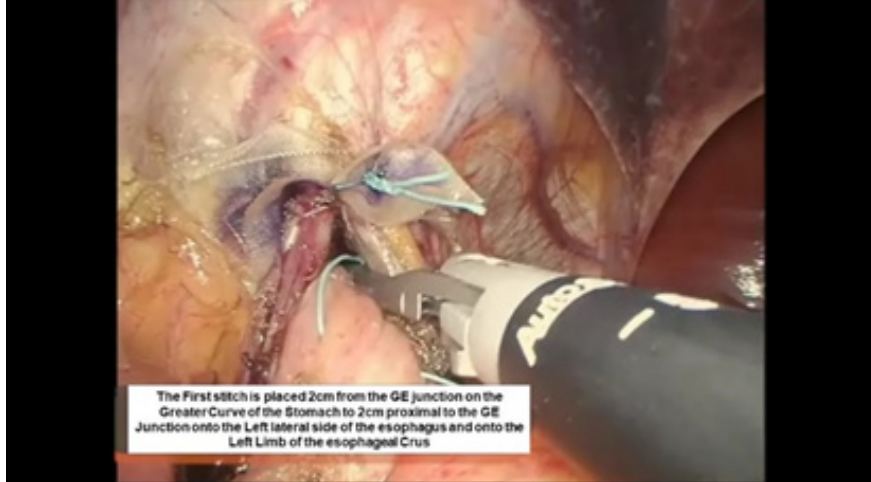

Figure 5: First belsey suture placement G1 to E1.

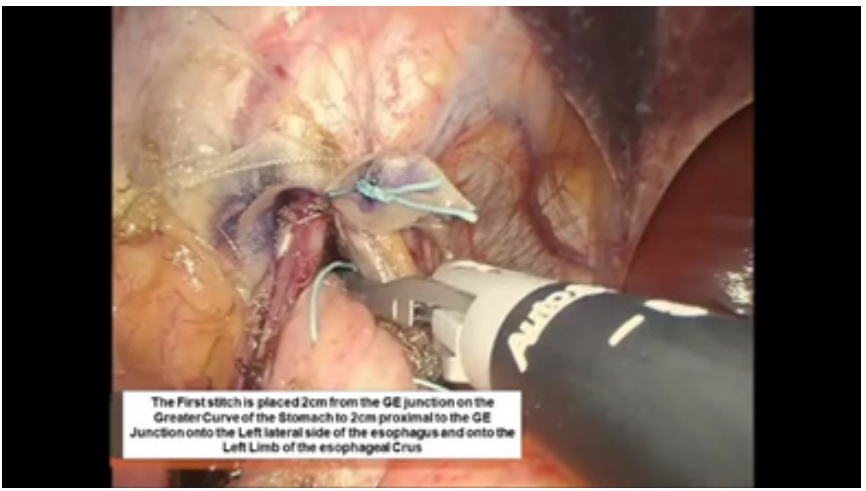

Figure 6: First belsey suture placement G1 to E1.

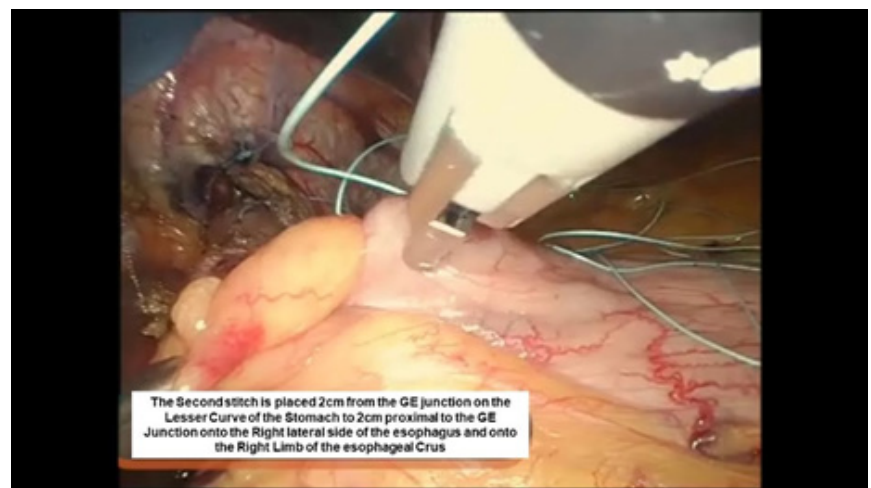

Figure 7: Second belsey suture placement, G2 to E2.

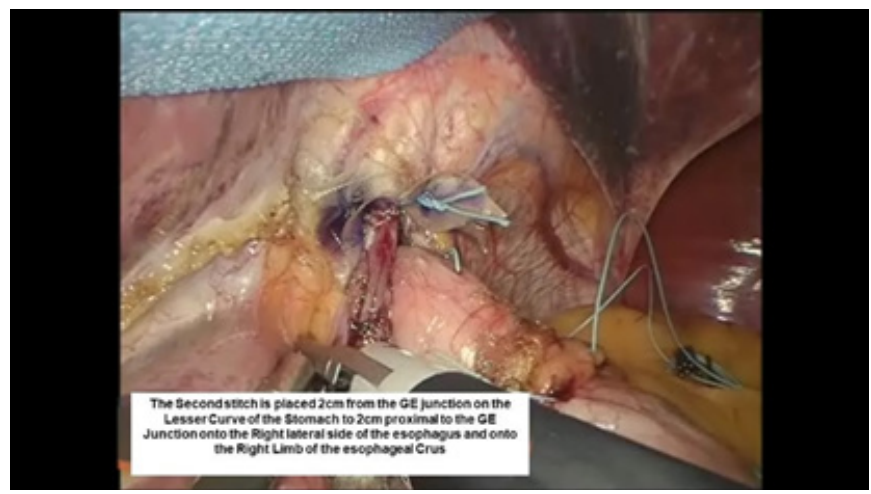

Figure 8: Second belsey suture placement, G2 to E2.

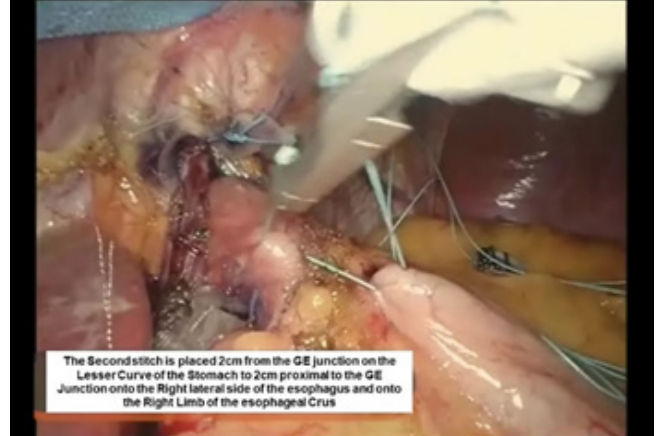

Figure 9: Second belsey suture placement, G2 to E2.

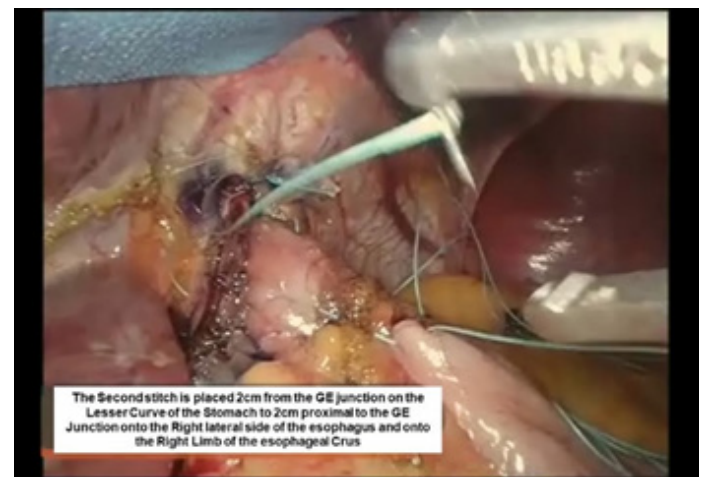

Figure 10: Second belsey suture placement, G2 to E2.

The third Belsey suture (E2 to G2, midpoint) is introduced in the same manner from G2 to E2 and through the diaphragm at the midpoint of the crural arch. This suture is withdrawn from Port 5 and tied using a knot-pusher and extracorporeal knots. Next, the "E1to G1" suture is withdrawn out of Port 5 and tied. Finally, the "E3 to G3" suture is withdrawn out of Port 5 and tied (Figure 11-13). Placement of the mattress Belsey sutures results in the intussusception of the esophagus into the stomach by $2 \mathrm{~cm}$ for 270 degrees (Figure 14,15). Only the camera port needs to be closed. This trocar site is closed using a laparoscopic suture passer and 0 Vicryl (Ethicon Endo-Surgery). $\mathrm{CO}_{2}$ is evacuated from the highest trocar by placing the patient in a steep reverse Trendelenburg position. The other Vertiport trocars are removed, and the tissues are allowed to close around the introducer sheath. Subcutaneous tissues are closed with 00 Vicryl, and the skin is closed with staples.

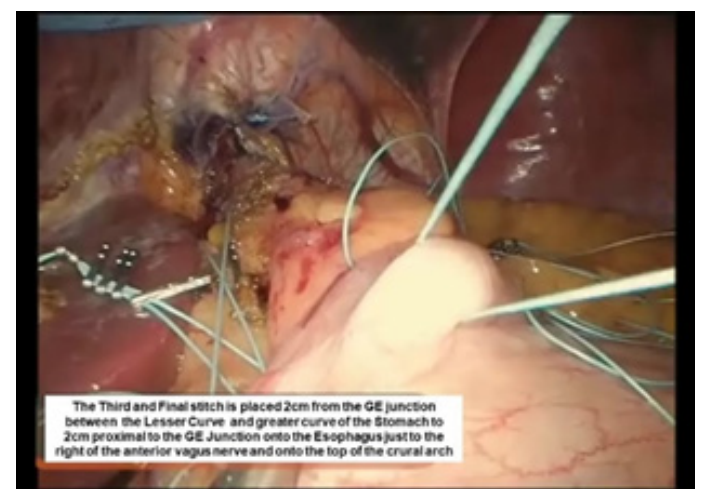

Figure 11: Third belsey suture placement, G2 to E2. 


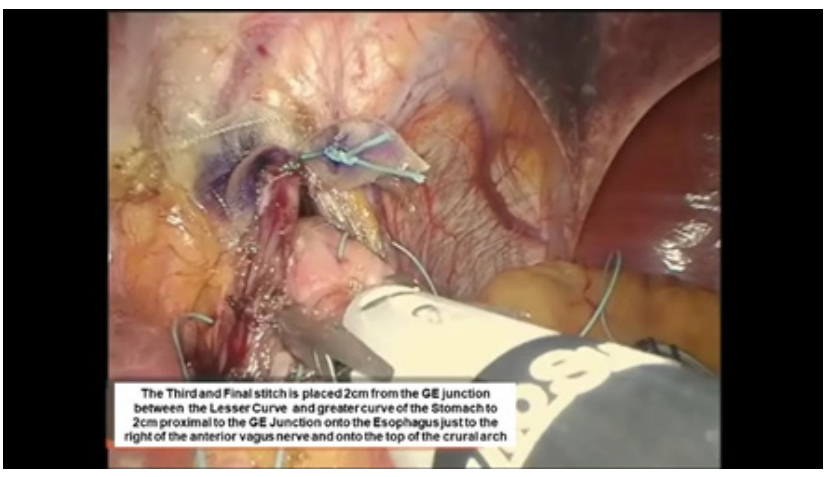

Figure 12: Third belsey suture placement, G2 to E2.

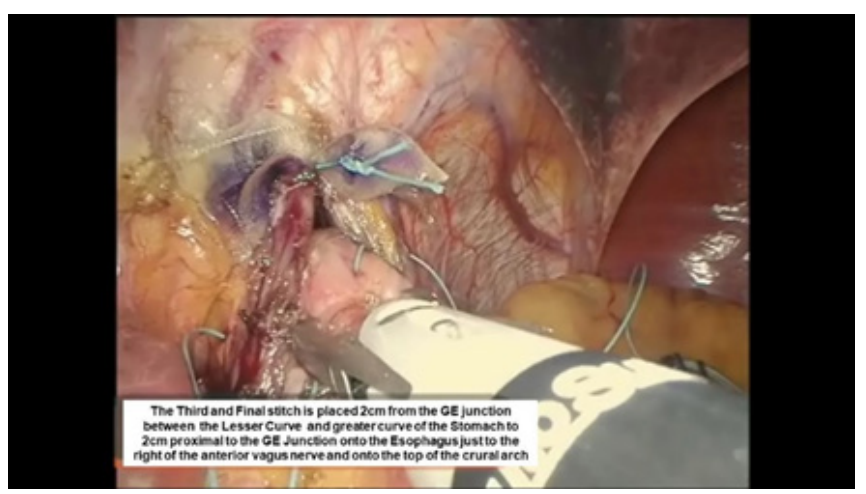

Figure 13: Third belsey suture placement, G2 to E2.

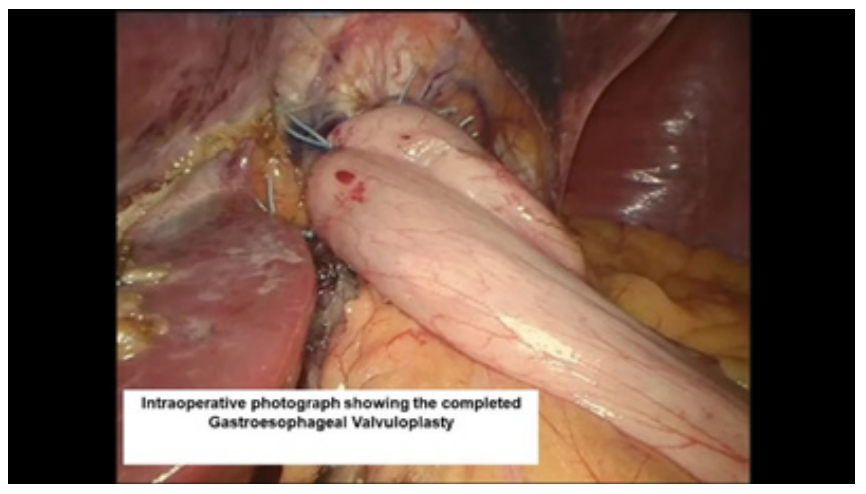

Figure 14: Intraoperative photograph of the completed belsey fundoplasty.

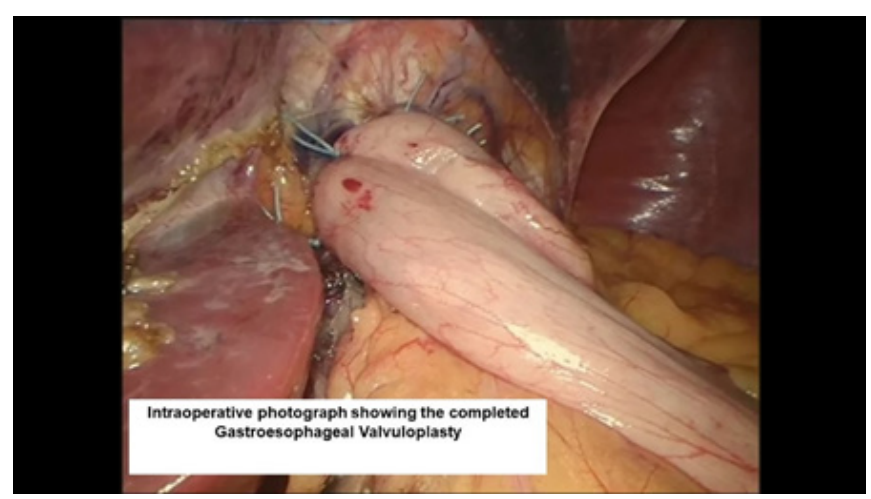

Figure 15: Endoscopic view of the completed belsey fundoplasty.
Result

During a 71-month period, 302 patients underwent robotic GE valvuloplasty. Eleven patients (3.6\%) were lost to follow up. In the remaining 291 patients, there were 156 men and 135 women. The mean age was $51+/-14$ years. Indication for surgery was failure of medical therapy in $212 / 291$ patients (73\%) and upper respiratory symptoms (cough, hoarseness, bronchospasm) in $79 / 291$ patients (27\%). On upper GI endoscopy, 183/291 patients (63\%) had a Hill Grade IV GE junction, 73/291 (25\%) had a Hill Grade III GE junction, and the reaming 35/291 (12\%) patients were graded as Hill Grade II. On contrast esophagography, $230 / 291$ patients $(79 \%)$ were diagnosed with a hiatal hernia. All patients had normal esophageal motility and increased acid exposure. At the time of surgery, exploration of the esophageal hiatus revealed a hiatal defect in 269/302 (87\%) patients. The mean operative time was $130+/-52$ minutes. In 19/291 patients $(6 \%)$, the intraoperative post-fundoplication endoscopy revealed an incompetent gastroesophageal valve. In these patients, the valve underwent further repair in order to obtain a satisfactory Hill Grade I score. Complications were seen in 61 patients (21\%). The pleura was entered in 55/291 (19\%) patients. This was treated with closure of the pleural opening and intraoperative evacuation of the pleural space. There was no conversion to an open procedure. A pneumothorax was diagnosed in 5/291 patients (1.7\%) postoperatively. These patients underwent drainage with a 10-French radiographically placed pig tail catheter. One patient had atrial fibrillation $(0.3 \%)$. There was no mortality. Mean hospitalization was $2.8+/-1.7$ days (median 2 days). Early (1-12 Weeks) Postoperative Results: Immediately after surgery, 221/291 patients $(76 \%)$ reported dysphagia to solids. Dysphagia had resolved in all patients by the third postoperative week. There was no incidence of gas bloat in the early postoperative period. By 12 weeks, acid suppression therapy was discontinued in all patients. Of the 291 patients, 5 patients (2\%) had transient gastroparesis, which resolved by the third postoperative month.

\section{Late follow-up}

Mean follow-up was $85+/-7$ months. At the time of follow up, the mean score on the SSQ decreased from 8.3+/- 0.6 to $0.7+/-0.2$ $(\mathrm{p}<0.05)$ Of the 291 patients, 279 patients $(96 \%)$ scored 0 on the questionnaire and were completely asymptomatic. The remaining patients (4\%) had some degree of heart burn and continued acid suppression therapy. Longterm gas bloat was not reported by any patient. Preoperatively 58/291 patients were objectively graded as Visick III, and 231/291 patients (80\%) were Visick IV. At the time of follow-up, 276/291 patients (95\%) were graded as Visick I and $5 \%$ as Visick II. The hiatal hernia recurred in $8 / 291$ patients (2\%). Recurrence was documented on upper GI endoscopy and contrast esophagography. In all instances, the recurrence was in the anterior aspect of the esophageal hiatus. There was no posterior crural disruption.

\section{References}

1. Locke GR, Talley NJ, Felts SL (1997) Prevalence and clinical spectrum of gastroesophageal reflux disease: A population based study in Olmsted county, Minnesota. Gastroenterology 112(5): 1448-1450. 
2. DeMeester TR, Wang CI, Weraly JA, et al. (1980) Technique, indications and clinical use of 24-hour $\mathrm{pH}$ monitoring. J Thorac Cardiovasc Surg 79(5): 656-670.

3. Souba WW (2000) Links in the chain: Gastroesophageal reflux disease, barrett's esophagus, dysphasia, and carcinoma. Contemporary Surg 56: 57-58.

4. Richter JE (2000) Chest pain and gastroesophageal reflux disease. J Clin Gastrownterol 30(3 Suppl): S39-S41.

5. Devesa SS, Blot WJ, Fraumeni JF Jr (1998) Changing patterns in the incidence of esophageal and gastric carcinoma in the United States. Cancer 83(10): 2049-2053.

6. DeMeester TR, Bonavina L, Albertucci M (1986) Nissen fundoplication for gastroesophageal reflux disease: Evaluation of primary repair in 100 consecutive patients. Ann Surg 204(1): 9-20.

7. Spechler SJ (2001) Screening and surveillance for complications related to gastroesophageal reflux disease. Am J Med 111(Suppl 8A): 130S-136S

8. Peters JH, DeMeester TR, Crookes P, Oberg S, de Vos Shoop M, et al. (1998) The treatment of gastroesophageal reflux disease with laparoscopic
Nissen fundoplication: Prospective evaluation of 100 patients with typical symptoms. Ann Surg 228(1): 40-50.

9. Lerut T, Coosemans W, Christiaens R, Gruwez JA (1990) The belsey mark iv antireflux procedure: Indications and long-term results. Acta Gastroenterol Belg 53(5-6): 585-590.

10. Gharagozloo F, Pourshahmir M, Deters K (1998) Comparison of nissen fundoplication and gastroesophageal valvuloplasty in an experimental model of gastroesophageal reflux disease. Surg Forum 10: 73-86

11. Hill LD, Kraemer SJM, Aye RW, et al. (1994) Laparoscopic hill repair. Gastrointest Endosc 40(2 Pt 1): 13-20.

12. Hansdotter I, Björ O, Andreasson A, Agreus L, Hellström P, et al. (2016) Hill classifcation is superior to the axial length of a hiatal hernia for assessment of the mechanical anti-reflux barrier at the gastroesophageal junction. Endosc Int Open 4(3): E311-E317.

13. Gharagozloo F, Soberman M, DuBree K, Tempesta B, Gurfnchel G (1999) Correlation of a hiatal hernia and gastroesophageal refux disease: A prospective blinded study in patients undergoing laparoscopy. Chest 116(4): 371.

For possible submissions Click below: 